\title{
Etude hydrobiologique d'un cours d'eau du Moyen-Atlas : l'oued Tizguit (Maroc)
}

\author{
M. Touabay ${ }^{1}$ \\ N. Aouad ${ }^{1}$ \\ J. Mathieu ${ }^{2}$
}

Mots-clés : Oued Tizguit, Moyen-Atlas, paramètres physico-chimiques, inventaire faunistique, richesse spécifique, évolution temporelle, variation longitudinale.

Le but de cette étude est de mieux connaître la qualité de l'eau et la macrofaune benthique des cours d'eau marocains du Moyen-Atlas. L'Oued Tizguit a été choisi pour son accessibilité et la variété de son environnement. Onze stations ont été suivies en mesurant 8 paramètres physico-chimiques et en faisant l'inventaire faunistique pendant un cycle hydrologique.

Ce travail montre que la qualité de l'eau évolue dans le temps selon un gradient amont-aval.

Des prélèvements qualitatifs ont permis de dénombrer 167 taxons appartenant à différents groupes où les Coléoptères sont majoritaires. La distribution de cette macrofaune semble déterminée par la variation longitudinale des différents paramètres du milieu, et sa composition doit son originalité, en partie, à la qualité de l'eau du milieu.

\section{Hydrobiological study of a river from the Moyen-Atlas : the Tizguit wadi (Morocco)}

Keywords : Tizguit wadi, Moyen-Atlas, physical and chemical factors, fauna inventory, species richness, changes with time, longitudinal changes.

The aim of this study is to gain a better understanding of the water quality and the benthic macrofauna of Moroccan rivers from the Moyen-Atlas. The Tizguit wadi was chosen for its accessibility and its environmental variability. Eight physical and chemical factors were measured and the fauna inventory was made at 11 sites during an hydrological cycle.

The results showed that changes occurred along an upstream-downstream gradient.

The number of sampled taxa was 167 belonging to different groups, where Coleoptera were dominant. The distribution of this fauna depends mainly on the environmental factors, and the originality of its composition depends on the water quality.

\section{Introduction}

Le Maroc, grâce à ses façades atlantique et méditerranéenne, possède l'un des plus important et des plus diversifié réseau hydrographique du nord de l'Afrique. Ses eaux continentales, de par leur richesse et leur diversité, ont suscité l'intérêt de chercheurs, dont Beauchamp (1949), Iablokoff (1954), Aouad (1984, 1991),

1. Université Sidi Mohamed Ben Abdellah, Faculté des Sciences Dhar El Mehrez- B.P. 1796 Atlas, Fès (Maroc).

2. Université Claude Bernard Lyon I, Hydrologie et Ecologie Souterraines, U.M.R. C.N.R.S. 5023, 43 Bd du 11 novembre 1918 , 69622, Villeurbanne cedex (France).
Giudicelli \& Dakki (1984), Dakki (1986 a, 1987), Essafi et al. (1993). Malheureusement, peu de travaux ont fait l'objet de suivis, tant au niveau des variations des facteurs d'environnement que des facteurs biologiques (Badri 1985, Benhoussa et al. 1988, Dakki 1985 a, 1985 b, 1986 b, Qninba et al. 1988, El Agbani et al. 1992). Certains concernent la qualité de l'eau sur des plans physico-chimiques et bactériologiques (Chahlaoui et al. 1997), mais la plupart sont des travaux non publiés.

Ce travail réalisé sur l'oued Tizguit est original, dans la mesure où aucune recherche hydrobiologique n'y avait été faite auparavant, et il contribue à une meilleure connaissance des cours d'eau du Moyen-Atlas marocain. Il a donc semblé intéressant d'axer ces investiga- 
tions sur les composantes abiotiques et leur évolution, de façon à déterminer leur influence sur la composition et la structure des peuplements benthiques. La prise en considération approfondie de la faune aquatique, par l'étude de la structure des peuplements devrait permettre une meilleure connaissance du milieu et une évaluation plus précise de la qualité de cet écosystème aquatique.

\section{Présentation du milieu d'étude et choix des stations}

\subsection{Localisation géographique}

Le Moyen-Atlas d'orientation Nord-Est, Sud-Ouest constitue l'élément central de l'ensemble montagneux marocain. Il est limité au nord par le couloir sud-rifain qui le sépare de la chaîne du Rif, au sud par le Haut-Atlas et la haute Moulouya, à l'est par la vallée de la moyenne Moulouya et à l'ouest par le plateau central.

L'oued Tizguit (Fig. 1) se situe dans le causse moyen-atlasique, et constitue le cours supérieur de l'oued Mikkes (Zeryouhi 1977). Le bassin versant, d'une superficie de $475 \mathrm{~km}^{2}$ est constitué essentiellement de formations calcaires et dolomitiques, d'âge liasique, qui reposent sur le Trias argileux et basaltique (Colo 1960-61). Il se situe dans l'étage bioclimatique humide. Le climat est montagnard de type méditerranéen, avec un été chaud et sec et un hiver froid et pluvieux. Son bassin versant est peuplé de cédraies denses, de pelouses et de steppes à chamaephytes ligneux. La ripisylve (peupliers, saules...) est importante et borde le cours d'eau sur pratiquement tout son parcours.

\subsection{Description des stations}

Onze stations situées le long de l'oued Tizguit (Fig. 1) ont été retenues pour cette étude. Leurs caractéristiques sont regroupées dans le tableau 1.

- Les stations SS0, SS1 et SS2, localisées les plus à l'amont du système, sont temporaires. Elles sont situées dans une zone caractérisée par l'absence totale de végétation et par la forte perméabilité du terrain. Leur mise en eau, de courte durée (décembre à juin), est assurée par les fortes précipitations de l'hiver et par la remontée de la nappe phréatique. Ces stations, et particulièrement la source (SS0) qui sort du massif karstique, sont très fréquentées par la population rurale.

- La station $\mathrm{S} 1$ se situe à l'aval immédiat de la confluence de l'oued Tizguit avec l'Oued Bousraf. La végétation environnante y est très développée et la couverture forestière enchevêtrée.
- La station S2, dont les eaux sont strictement d'origine souterraine, est une résurgence karstique de la nappe phréatique de l'oued Tizguit. Le lit est de faible largeur. Il est tapissé de cailloux rugueux, de graviers et de galets.

- La station S3 est profonde, aux eaux calmes, et se situe à $16,5 \mathrm{~m}$ de la résurgence. De fortes averses, survenues fin novembre 1996, ont provoqué un lessivage des rives et rendu les eaux de cette station très boueuses et troubles.

- La station S4 se caractérise par une végétation aquatique et riveraine diversifiée et abondante. Au niveau de cette station, l'oued Tizguit reçoit un affluent karstique qui provient de la source Ain Termilat.

- La station S5 est située juste en aval de la ville d'Ifrane et en reçoit les eaux résiduaires. Les eaux sont troubles, de couleur noirâtre et ont une faible vitesse d'écoulement.

- La station S6 est située en bordure de la route Ifrane-El Hajeb. C'est un bras mort peu profond, de faible vitesse de courant, et très ensoleillé en raison de l'absence de couverture forestière.

- La station S7, au confluent du ruisseau provenant de la source Vittel avec l'oued Tizguit, est soumise à une influence touristique particulièrement intense en été.

- La station S8, au confluent de l'oued Tizguit avec l'oued Zerrouka, est une zone très ombragée par suite de la présence de vieux peuplements forestiers.

\section{Matériel et méthodes}

\subsection{Paramètres physico-chimiques}

Une étude des paramètres physico-chimiques de l'eau a été entreprise au niveau de chacune des stations, dans le but de connaître les modifications de sa qualité, en fonction de son origine (épigée ou souterraine, ou de son mélange), et du temps. Pour chaque station, les paramètres suivants ont été mesurés mensuellement d'avril 1996 à mars 1997 : température de l'air et de l'eau, $\mathrm{pH}$, conductivité et minéralisation, oxygène dissous, teneurs en $\mathrm{Ca}^{2+}, \mathrm{Mg}^{2+}$, alcalinité $\left(\mathrm{HCO}^{3-}\right)$ et dureté totale (THT). Les mesures ont été effectuées au laboratoire sur des échantillons d'eau de $500 \mathrm{ml}$ placés dans une boîte isotherme.

Le dosage de l'oxygène dissous est effectué par la méthode de Winkler (Rodier 1996), l'oxygène étant fixé sur le terrain, et la teneur en $\mathrm{O} 2$ est exprimée en $\mathrm{mg} / \mathrm{l}$.

Le pH est mesuré à l'aide d'un électro-pH-mètre (modèle WTW PH/SET-1). 


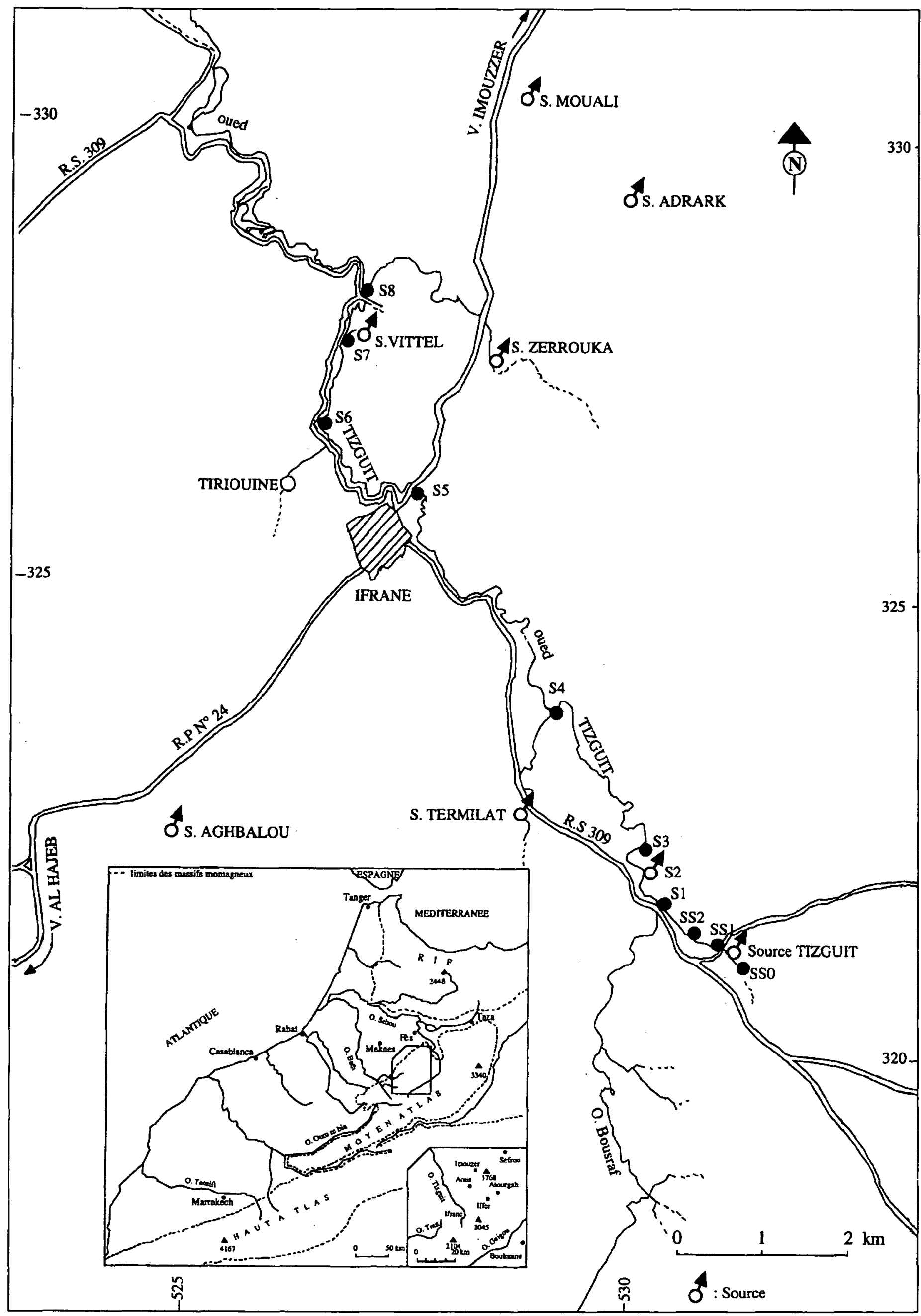

Fig. 1. Localisation des stations d'étude le long de l'oued Tizguit Fig. 1. Location of the sites along the oued Tizguit. 


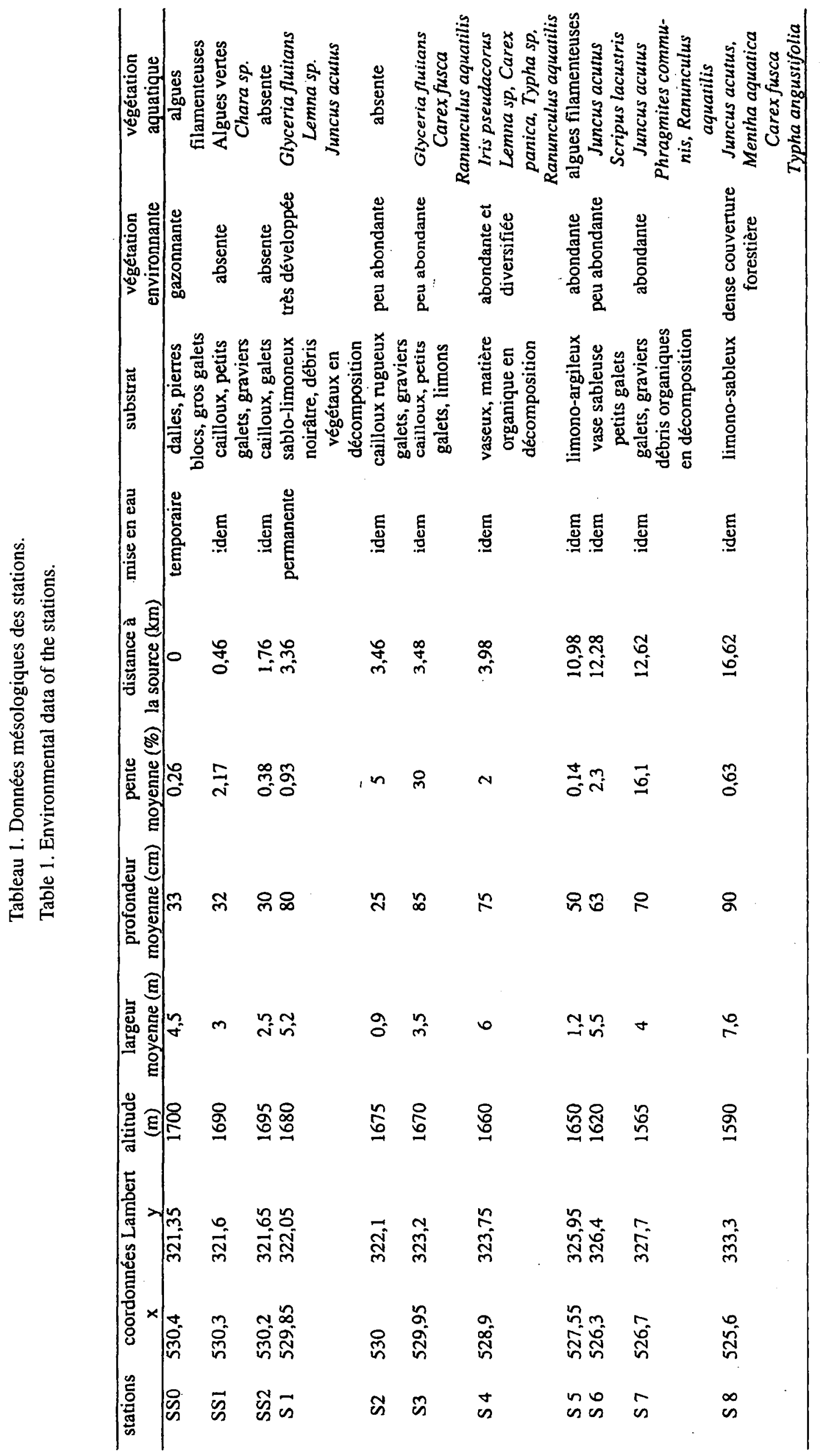


La conductivité est mesurée à l'aide d'un conductimètre (modèle WTW LF 318/SET) et exprimée en $\mu$ S.cm à une température de $20^{\circ} \mathrm{C}$.

La dureté totale ou teneur en alcalino-terreux (concentration totale en ions calcium, magnésium et autres ions bivalents et trivalents exprimée en $\mathrm{mg} / \mathrm{l}$ ) a été déterminée par titrimétrie à l'EDTA (Rodier 1996).

L'alcalinité (TAC) a été mesurée avec la méthode décrite par Rodier (1996). Elle est exprimée en mg/l de $\mathrm{HCO}^{3-}$.

\subsection{Prélèvements de faune}

Les prélèvements de faune ont été effectués au filet troubleau. Au niveau de chaque station, et afin de récolter le maximum d'espèces, chaque échantillon est constitué de trois prélèvements élémentaires suivant un transect réalisé d'une rive à l'autre (à l'exception de la station S3, trop profonde). Les résultats des trois prélèvements élémentaires sont globalisés dans les différentes analyses.

Comme pour les mesures physico-chimiques, les récoltes de faune ont été faites mensuellement, du mois d'avril 1996 au mois de mars 1997. Les stations temporaires situées le plus en amont (SS0, SS1, SS2) n'ont pu être prélevées lors de la période d'assec, de juillet à novembre 1996 . Une crue survenue fin oc- tobre, début novembre 1996, a empêché tout prélèvement dans les stations situées à l'aval.

Les résultats semi-quantitatifs sont exprimés comme suit : 1 = nombre moyen d'individus d'une espèce récoltée lors de la campagne compris entre 1 et 5 ; $2=$ entre 6 et $10 ; 3=$ entre 11 et $20 ; 4>21$.

\subsection{Analyse statistique}

Les données physico-chimiques et faunistiques ont été traitées par analyses en composantes principales et analyses factorielles des correspondances avec le logiciel ADE (Analysis of Environnemental Data) de Thioulouse et al. (1997)

\section{Résultats}

\subsection{Paramètres physico-chimiques}

Les variations temporelles et stationnelles des composantes physico-chimiques sont représentées sur les figures 2 et 3 .

L'analyse en composantes principales permet de faire ressortir un gradient amont - aval sur le plan F1 - F2 (les deux premiers facteurs $\mathrm{F} 1$ et $\mathrm{F} 2$ expliquent $89 \%$ de la variabilité totale). Il tend à séparer sur l'axe F1 les
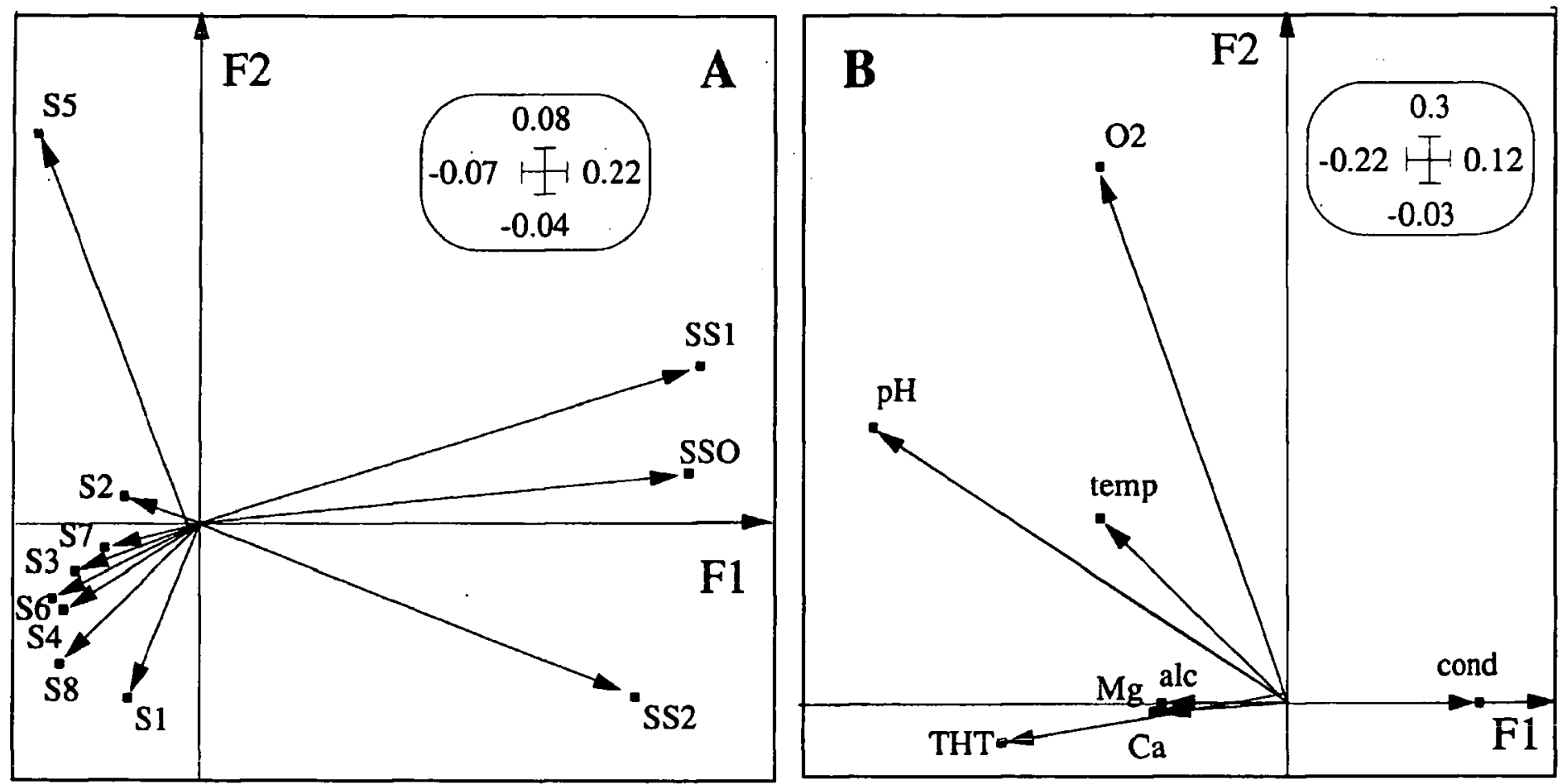

Fig. 2. Analyse en Composantes Principales des paramètres physico-chimiques. Plan factoriel F1-F2. A : carte des stations (SS0 à S8), $\mathrm{B}$ : carte des paramètres écologiques. temp $=$ température, $\mathrm{pH}, \mathrm{O} 2=$ oxygène dissous, cond $=$ conductivité, alc $=\mathrm{al}-$ calinité, $\mathrm{THT}=$ dureté totale, $\mathrm{Mg}=$ magnésium, $\mathrm{Ca}=$ calcium

Fig. 2. Principal Components Analysis on the physical and chemical parameters. F1-F2 plane of the factorial maps of sampling sites (A) and ecological parameters (B) SS0 to S8 represent the stations. temp = temperature, $\mathrm{pH}, \mathrm{O} 2=$ dissolved oxygen, cond $=$ conductivity, alc $=$ alkalinity, $\mathrm{THT}=$ total hardness, $\mathrm{Mg}=$ Magnesium, $\mathrm{Ca}=$ Calcium. 


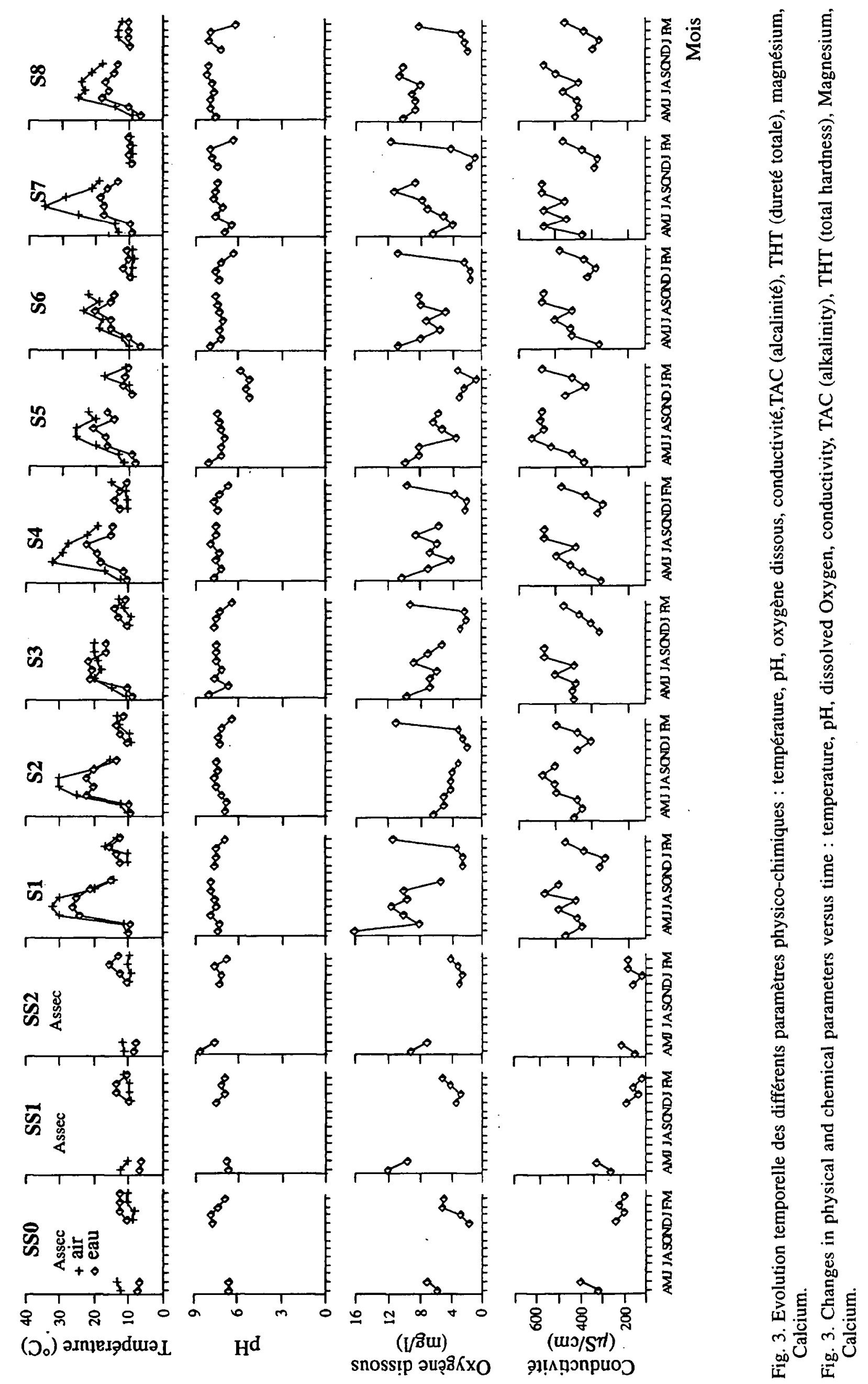




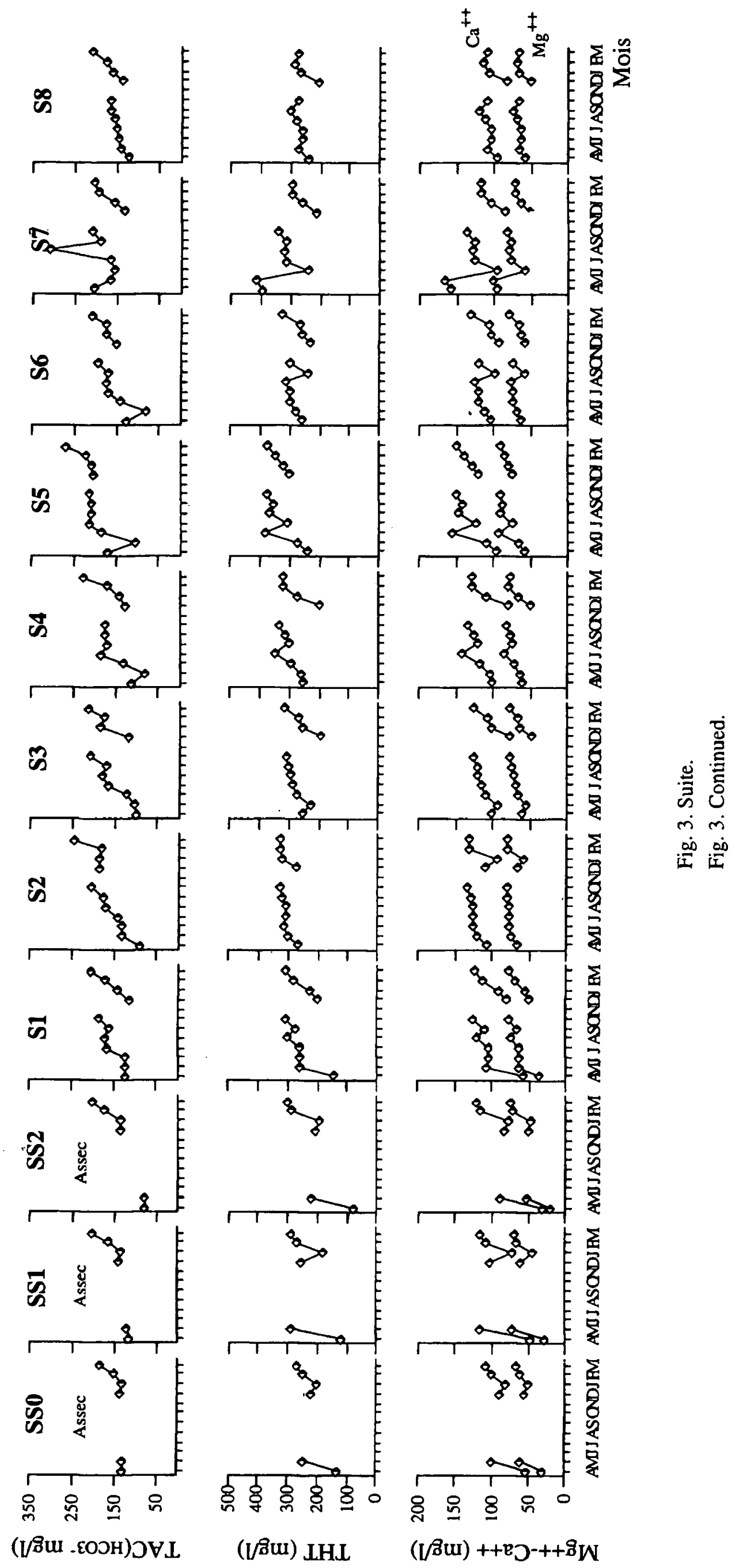


stations temporaires situées les plus à l'amont, et dont l'ensemble des valeurs des paramètres sont plus faibles que celles des autres stations (en particulier la conductivité). L'axe F2 sépare la station S5, caractérisée par une faible teneur en oxygène dissous et un faible $\mathrm{pH}$, située à l'aval de la ville d'Ifrane (Fig. 2) des autres stations.

L'analyse spatio-temporelle des différents paramètres permet d'expliquer ces résultats (Fig. 3 ) :

\section{1 - Régime thermique}

Les variations saisonnières de la température de l'eau suivent celles de l'air, avec des maxima à la fin du printemps et en été, mais présentent de plus faibles amplitudes.

La température de l'eau est plus faible dans les stations temporaires (SS0, SS1 et SS2 : entre $6^{\circ}$ et $13^{\circ} \mathrm{C}$ ), pour lesquelles il n'a pas été possible de faire de mesures en été, que dans les stations permanentes (entre $8^{\circ}$ et $20^{\circ} \mathrm{C}$ ). Le maximum a été enregistré en juin, juillet et août dans la station $S 1\left(26^{\circ} \mathrm{C}\right)$ malgré un important couvert végétal. Cette valeur importante est due aux apports de l'oued Bousraf dont les eaux ont une température plus élevée.

$2-\mathrm{pH}$

Le $\mathrm{pH}$ est voisin de la neutralité (entre 7 et 8 ) dans toutes les stations à l'exception de S5, pour laquelle il est de l'ordre de 5, de décembre 1996 à mars 1997. L'acidité de l'eau de cette station, en cette période, est certainement due aux eaux résiduaires qui y sont déversées.

\section{3 - Oxygène dissous}

En SSO, la teneur est de l'ordre de $6-8 \mathrm{mg} / \mathrm{l}$, valeur élevée, voisine de la saturation, comparée aux valeurs données pour les sources karstiques par Nisbet \& Verneaux (1970).

Les variations saisonnières de la teneur en $\mathrm{O}_{2}$ dissous dans l'eau des stations sont très marquées.

Au printemps, suite à une production accrue-(en particulier par la prolifération d'algues filamenteuses), la teneur atteint des valeurs importantes : jusqu'à $16 \mathrm{mg} / \mathrm{l}$ en avril 1996, à la station S1. C'est dans la station S5 qu'elle est la plus basse.

Au début de l'hiver, curieusement, les valeurs observées sont peu élevées et comprises entre 1 et $4 \mathrm{mg} / 1$

\section{4 - Conductivité}

Proportionnelle à la quantité des sels ionisables dissous, elle constitue une bonne indication du degré de minéralisation d'une eau. D'après les classes proposées par Nisbet \& Verneaux (1970), l'eau de l'oued Tizguit passe d'une minéralisation moyenne (200 à 400 $\mu \mathrm{S} / \mathrm{cm}$ ) dans les stations temporaires situées en amont (SS0, SS1, SS2) à une minéralisation plus élevée (300 à environ $600 \mu \mathrm{S} / \mathrm{cm}$ ) dans les stations permanentes (S1 à S8). Les variations sont saisonnières, avec maxima au printemps et en été.

5 - Duretés totale (THT), calcique et magnésienne

La dureté totale est comprise entre 80 et $300 \mathrm{mg} / 1$ dans les stations SS0, SS1 et SS2, entre 140 et 300 $\mathrm{mg} / 1$ dans $\mathrm{S} 1$, S2 et $\mathrm{S} 3$ et entre 150 et $420 \mathrm{mg} / 1$ dans les autres stations S4 à S8. Ce paramètre présente des valeurs, échelonnées sur les trois dernières classes $(4,5$, 6) de Nisbet \& Verneaux (1970), qui correspondent à des eaux de plus en plus dures de l'amont vers l'aval, sans qu'il existe de véritable gradient, particulièrement en saison estivale où la solubilité du $\mathrm{CO}_{2}$ diminue lorsque la température est plus élevée.

La dureté calcique varie de 32 à $100 \mathrm{mg} / \mathrm{l}$ dans SSO, $\mathrm{SS} 1$ et $\mathrm{SS} 2$, de 50 à $125 \mathrm{mg} / 1$ dans $\mathrm{S} 1, \mathrm{~S} 2$ et $\mathrm{S} 3$ et de 80 à $170 \mathrm{mg} / \mathrm{l}$ dans $\mathrm{S} 4$ à $\mathrm{S} 8$. Ces valeurs élevées sont dues aux calcaires du Jurassique qui constituent le substrat principal du bassin versant de l'oued Tizguit.

La teneur en magnésium évolue de façon identique à celle du calcium, avec cependant des valeurs plus faibles dans les stations temporaires $(20$ à $70 \mathrm{mg} / \mathrm{l})$ et légèrement plus élevées dans les stations permanentes $(50$ à $100 \mathrm{mg} / \mathrm{l})$. Le magnésium est essentiellement dû à la présence de calcaires dolomitiques dans le bassin versant.

\section{6 - Alcalinité (TAC)}

Les valeurs de ce paramètre augmentent globalement de l'amont vers l'aval. Elles varient de 75 à 270 $\mathrm{mg} / \mathrm{l}$ et sont conformes à celles trouvées dans les régions calcaires, comme le Jura, où les valeurs sont généralement comprises entre 100 et $250 \mathrm{mg} / \mathrm{l}$ (Rodier 1996).

\subsection{Etude faunistique}

Un inventaire, le plus exhaustif possible, de la faune du cours d'eau a été réalisé ainsi qu'un essai de suivi de la dynamique des peuplements.

\subsubsection{Analyse globale}

Le plan factoriel F1 - F2 de l'analyse des correspondances (Fig. 4) fait apparaître sur l'axe F1 l'opposition entre les stations situées à l'amont de la ville d'Ifrane (S1 à S4) et les stations S5 à S8 à l'aval (la station S5, située immédiatement à l'aval d'Ifrane étant la plus éloignée de la station $\mathrm{S} 1$ ), les deux premiers facteurs F1 et F2 expliquent $53 \%$ de la variabilité totale. Sur l'axe F2, les stations temporaires de l'amont, SS0 à 


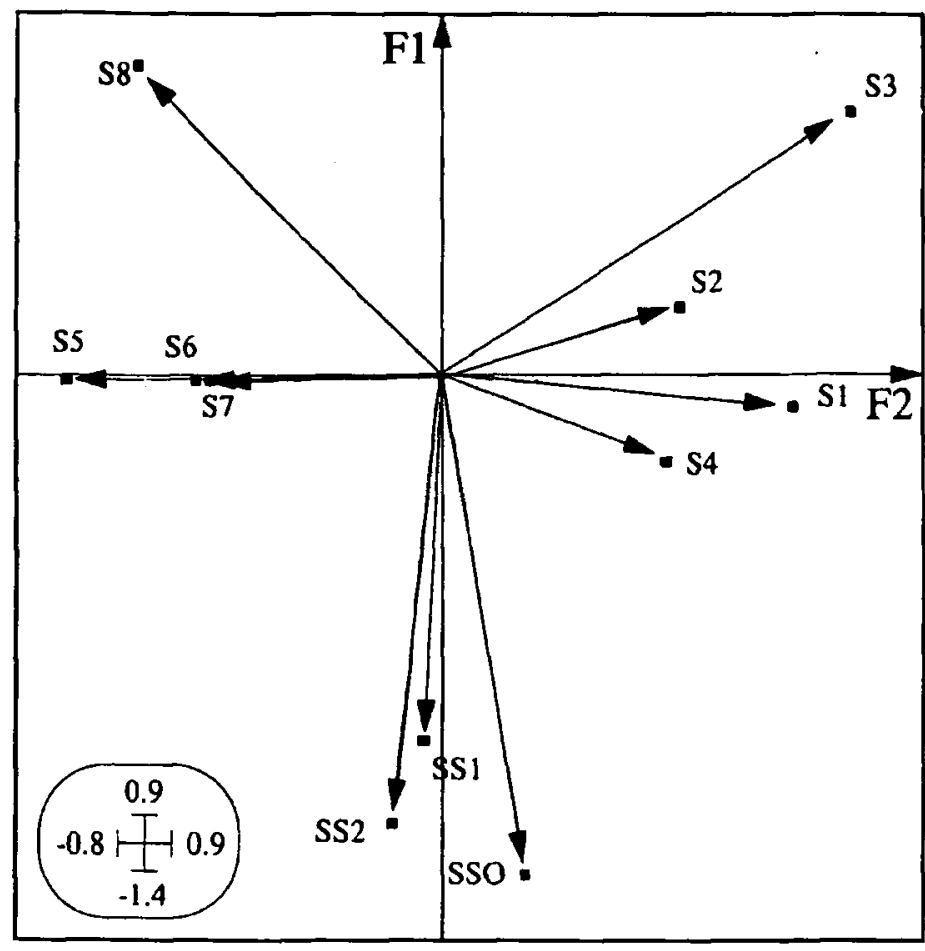

Fig. 4. Analyse Factorielle des Correspondances sur les données biologiques. Plan F1-F2. SS0 à S8 : code des stations.

Fig. 4. Correspondance Analysis of the biological data. F1-F2 plane of the factorial map. SSO to S8 : represent the stations.

SS2, s'opposent aux stations pérennes de l'aval. Cette organisation s'explique par l'étude de l'inventaire faunistique et de la richesse spécifique, ainsi que par l'évolution temporelle du peuplement.

\subsubsection{Inventaire faunistique et richesse spécifique glo- bale}

Les prélèvements semi-quantitatifs ont permis de recenser 167 taxons pour lesquels, dans la mesure du possible, la détermination a été faite jusqu'à l'espèce, à l'exception des Diptères et des Arachnides, déterminés seulement à la famille.

Ces taxons sont constitués, par ordre de richesse spécifique décroissante (Tableau 2), de Coléoptères (54), Hétéroptères (27), Mollusques (23), Diptères (17), Ephéméroptères (16), Trichoptères (7), Annélides (6), Odonatoptères (6), Crustacés (4), Arachnides (4) et Plécoptères (4).

Dans le cours supérieur, on note une augmentation de la richesse spécifique de l'amont vers l'aval (Tableau 2) (11 espèces en SS0, 18 en SS1 et 28 en SS2) ainsi que du nombre de groupes : en SS0, 9 espèces sur 11 appartiennent à l'ordre des Coléoptères, puis apparaissent progressivement, en SS1 et SS2, d'autres groupes faunistiques, dont les Mollusques, les Hétéroptères, les Diptères et d'autres espèces de Coléoptères, comme les Hydrocanthares. Comparée au nombre total d'espèces recensées sur l'ensemble de l'oued (167), cette richesse reste faible. La pauvreté de ces trois stations est certainement la conséquence d'une végétation environnante et aquatique peu abondante, voire absente, d'un substrat caillouteux, et surtout d'une courte durée de mise en eau.

Lors de l'assèchement de ces stations en juin 1996, la prospection à l'intérieur du substrat a toutefois permis de récolter des larves d'Hydrophilidés (genre Helophorus), Hydrocanthares et Diptères. Une nouvelle prospection jusqu'à $-15 \mathrm{~cm}$, juste avant la remise en eau, n'a pas permis de trouver des organismes dans les sédiments devenus trop secs ; cet assèchement ne permet pas le déroulement normal du cycle vital.

Par rapport aux trois précédentes, la première station permanente de l'oued Tizguit (S1) montre une importante richesse spécifique (Tableau 2) : 75 taxons, dont la moitié sont des Coléoptères, notamment des Dytiscidés et des Hydrophilidés. L'oued Bousraf, qui se déverse dans l'oued Tizguit au niveau de cette station, l'enrichit très probablement par le phénomène de dérive (14 espèces exclusives).

Cet enrichissement de la faune par la dérive s'observe également au niveau des stations S4, S7 et S8 qui sont des points de confluence avec respectivement l'Aïn Termilet, la source Vittel et l'oued Zerrouka. En dehors de la station $\mathrm{S} 1$, elles présentent en effet elles aussi le plus grand nombre de taxons et d'espèces exclusives (respectivement 8 pour $S 4$ sur 66 taxons, 9 pour $\mathrm{S} 7$ sur 84 taxons et 10 pour $\mathrm{S} 8$ sur 70 taxons (Tableau 2). Ces stations constituent, en effet, des milieux favorables à l'installation d'une faune plus riche et plus diversifiée (en particulier Mollusques, Ephéméroptères, Hétéroptères et Coléoptères) grâce à une végétation environnante et aquatique abondante, un substrat riche en matière organique en décomposition.

La station S2, qui est alimentée directement par une résurgence karstique, est la moins diversifiée et la moins peuplée. On y rencontre principalement quelques Ephéméroptères, Hétéroptères, Coléoptères, mais pas de Trichoptères.

La station S3, la plus profonde, n'a pu faire l'objet d'un transect de 3 prélèvements unitaires d'une rive à l'autre. Elle est relativement diversifiée, et les taxons exclusifs sont essentiellement des Coléoptères inféodés aux eaux troubles.

La station S6, comparée aux stations situées plus en amont, présente une richesse spécifique plus faible (50 taxons), vraisemblablement en raison de sa configuration de bras mort, où la vitesse du courant est très faible, et de l'absence de végétation rivulaire. 
Tableau 2. Liste des taxons recensés dans les différentes stations de l'oued Tizguit. $1:$ moyenne du nombre d'individus d'une espèce récoltés lors de la campagne compris entre 1 et $5 ; 2:$ compris entre 6 et $10 ; 3:$ compris entre 11 et $20 ; 4: 4>21$.

Table 2. List of the taxa sampled in the stations of the Oued Tizguit. $1:$ species whose mean numbers of inviduals were between between 1 and $5 ; 2:$ those between 6 and $10 ; 3:$ between 11 and $20: 4>$ those above 21 .

\begin{tabular}{|c|c|c|c|c|c|c|c|c|c|c|c|c|}
\hline Stations & & SSO & SS1 & SS2 & S1 & s2 & $\mathbf{S 3}$ & S4 & S5 & S6 & S7 & $\mathbf{S 8}$ \\
\hline \multicolumn{13}{|l|}{ ANNELIDES } \\
\hline Glossiphomia complanata $\mathrm{L}$. & & & & & & & 1 & & 4 & & 1 & \\
\hline Helobdella stagnalis $\mathrm{L}_{. .}$ & & & & & & & 1 & & & 1 & & \\
\hline Haemopsis sangrasuga $\mathrm{L}$ & & & & & & & & 1 & 4 & & & \\
\hline Haplotoxis gordioides Hartmamn & & & 2 & 3 & 1 & & 2 & 2 & 4 & & 1 & 2 \\
\hline Tubificidae & & & & & & 3 & 4 & 1 & 4 & 1 & 2 & 1 \\
\hline$N$ taxons & 5 & 0 & 1 & 1 & 1 & 1 & 4 & 3 & 4 & 2 & 3 & 2 \\
\hline \multicolumn{13}{|l|}{ MOLLUSQUES } \\
\hline Theodoxus fluviatilis L.. & & & & 2 & & 1 & & 1 & 1 & 2 & 2 & 2 \\
\hline Gyraulus laevis Alder & & & & & & & & 1 & 1 & 1 & 2 & 2 \\
\hline Segmentina nitida Müllet & & & & & 1 & & 1 & 1 & 1 & 1 & 1 & 1 \\
\hline Succinea debilis Morelet & & & 1 & & 1 & 1 & 2 & 1 & 1 & 2 & 2 & 2 \\
\hline Melanopsis praemorsa L. & & & & & & & & & 1 & & 2 & \\
\hline Melanopsis castellata F. & & & & & & & & & 1 & 2 & 1 & 1 \\
\hline Mercuria confusa F. & & & & & & & & 1 & & 1 & 4 & \\
\hline Valvata piscinalis Müller & & & & & & & & & 1 & 2 & & \\
\hline Vatvata cristata Müller & & & & & 1 & & & 1 & 1 & 2 & 2 & 2 \\
\hline Ancylus fluviatilis Müller & & & & & & & & & & & & 1 \\
\hline Lymnaea peregra Müller & & & & & & & & & & & & 1 \\
\hline Lymnaea tnuncatula Müller & & & 2 & 2 & 2 & & & & 2 & 2 & 4 & 2 \\
\hline Lymnaea stagnalis L. & & & & & & & & & 1 & & 2 & 1 \\
\hline Physa acuta Drap. & & & & 2 & 2 & 2 & 2 & 3 & 2 & 2 & 4 & \\
\hline Planorbis planorbis $\mathrm{L}$.. & & & 2 & & 1 & 2 & 2 & 3 & 1 & 1 & 2 & 1 \\
\hline Anisus spirorbis L. & & & & & & & & & & & 1 & 1 \\
\hline Gyraulus cristala L.. & & & & & & & & & & 1 & 2 & \\
\hline Acroloxus lacustris $\mathrm{L}$. & & & & & & & & & & & 1 & \\
\hline Bulinus truncarus Audouin & & & & & & & & & 1 & & 1 & \\
\hline Ferrissia sp. & & & & & & & & & & & & 1 \\
\hline Pisidium sp. & & & & & & & & & 2 & 1 & & 1 \\
\hline Pisidium casertamum Poli & & & & & & & & & 1 & & & \\
\hline Margaritifera sp. & & & & & & & & & 2 & & & 1 \\
\hline $\mathrm{N}$ taxons & 23 & $\mathbf{0}$ & 3 & 3 & 6 & 4 & 4 & 8 & 16 & 13 & 16 & 15 \\
\hline \multicolumn{13}{|l|}{ ARACHNIDES } \\
\hline $\begin{array}{l}\text { Zodariides } \\
\text { Theridides }\end{array}$ & & & 1 & & 1 & & 1 & 2 & 2 & 2 & 1 & $\begin{array}{l}1 \\
1\end{array}$ \\
\hline Xysticus sp. & & & & & 1 & & 1 & & & & 1 & 1 \\
\hline Pholcius sp. & & & & & & & 1 & 2 & 2 & 1 & & 1 \\
\hline $\mathrm{N}$ taxons & 4 & 0 & 1 & 0 & 2 & 0 & 3 & 2 & 2 & 2 & 2 & 4 \\
\hline \multicolumn{13}{|l|}{ CRUSTACES } \\
\hline Gammanus pulex L. & & & & & & & & & & 3 & & \\
\hline Gammarus gouthieri P. & & & & & & & & & 4 & 4 & 4 & 4 \\
\hline Asellus aquaticus Geoffroy & & & & & & & 1 & & 1 & & & \\
\hline Apus producta Bosc. & & & & & & & & & 2 & & & \\
\hline $\begin{array}{r}\mathrm{N} \text { taxons } \\
\text { FPHEMFROPTERF }\end{array}$ & 4 & 0 & $\mathbf{0}$ & 0 & $\mathbf{0}$ & 0 & 1 & 0 & 3 & 2 & 1 & 1 \\
\hline \multicolumn{13}{|l|}{ EPHEMEROPTERES } \\
\hline $\begin{array}{l}\text { Potamanthus luteus L.. } \\
\text { Epeorus sylvicola Pictet }\end{array}$ & & & & & $\begin{array}{l}4 \\
2\end{array}$ & 2 & $\begin{array}{l}3 \\
2\end{array}$ & $\begin{array}{l}4 \\
1\end{array}$ & 1 & 2 & 3 & 2 \\
\hline $\begin{array}{l}\text { Epeorus sylvicola Pictet } \\
\text { Ectyonurus rothschildi Navas }\end{array}$ & & & & & 1 & 1 & 2 & 3 & $i$ & & 2 & 1 \\
\hline Ecdyonunus forcipula Pictet. & & & & & 1 & & & 1 & & & & \\
\hline Rhithrogena sp. & & & & & 1 & 1 & 1 & 2 & 2 & & & 1 \\
\hline Rhithrogena ayadi D.\& T. & & & & & 1 & & & 2 & 2 & & & \\
\hline Oligoneuriella skoura D.\& G. & & & & & & & & 2 & & & 1 & \\
\hline Oligoneuriopsis skhounate D.\&G. & & & & & & & & 2 & & & 1 & 1 \\
\hline $\begin{array}{l}\text { Baetis rhodani Pictet } \\
\text { Brachycercus sp. }\end{array}$ & & & & & 3 & & 2 & 1 & 1 & & & 1 \\
\hline Ephemera sp. & & & & & 2 & 1 & 2 & & & & 2 & 1 \\
\hline Ephemera glaucops Pictet & & & & & 1 & & & & & & 1 & 1 \\
\hline Ephemerella sp. & & & & & & & & 1 & & & & \\
\hline Ephemerella ignita Poda & & & & & & & & 1 & & & & \\
\hline $\begin{array}{l}\text { Prosopistoma sp. } \\
\text { Ephoron virgo Olivier }\end{array}$ & & & & & I & & & & 1 & & & \\
\hline $\mathrm{N}$ taxons & 16 & 0 & 0 & 0 & 10 & 5 & 6 & 12 & 7 & 1 & 6 & 7 \\
\hline
\end{tabular}


Tableau 2. Suite

Table 2. Continued.

\section{PLECOPTERES}

Brachyptero algirica Aubert Capnia sp.

Protonemoura algirica Aubert Perla marginata Panzer

ODONATES

$$
\mathrm{N} \text { taxons }
$$

Libellula quadrimaculata $\mathrm{L}$

Aeschna mixata Latr.

Anax imperator Leach

Gomphus simillimus Syls

Corchlegaster annularus Latr.

Lestes barbarus Fab.

HETEROPTERES

$$
N \text { taxons }
$$

Hebrus ruficeps Thomson

Mesovelia sp.

Mesovelia pygmae Duf

Hydrometra stagnorum $\mathrm{L}$

Velia ioannis Tamanini

Velia currens F.

Velia saulii Tamanini

Velia africana Tamanini

Velia rivulorum Fab.

Gerris lacustris L.

Gerris najas De Geer

Nepa cinerea L.

Nepa remyi Poisson

Nepa rubra rubra L.

Nepa rubra minor Puton

Naucoris macularus maculatus F.

Aphelocheirus aestivalis $\mathbf{F}$.

Notonecta pallidula Poisson.

Notonecta glonuca $\mathrm{L}$.

Notonecta maculata maculata $\mathrm{F}$.

Notonecta furcata $F$.

Notonecta obliqua meridionalis $P$.

Notonectn glauca nufescens $\mathbf{P}$.

Plea minutissima Fussl.

Sigara lateralis Leach

Corixa punctata Illiger.

Corixa affinis pallida Poisson

$$
N \text { taxons }
$$

COLEOPTERES

Agabus nitidus Fab.

Agabus nebulostus Forst.

Agabus didymus $\mathrm{Ol}$

Agabus chalconorus Panz

Agabus bigutarus Olivier

Agabus brunneus F.

Acilius sulcatatus L.

Colymbetes fuscus $\mathrm{L}$.

Ilybius fulginosus $\mathrm{F}$

Dytiscus marginalis L.

Laccophilus minitus L.

Cybister lateralimarginalis De Geer

Laccophilus hyalinus De Geer.

Stictonectes optatus Seill.

Hydroporus pulescens Gyll.

Hydroporus obsoletus Aube.

Hydropons discretus Fair.

Hydroparus Limbatus Aubé

Hydroporus nigrita Fab.

Hydroporus planus $\mathrm{Fab}$.

Hydroporus marginatus Duff.

Potamonectes grisiastritus De Geer

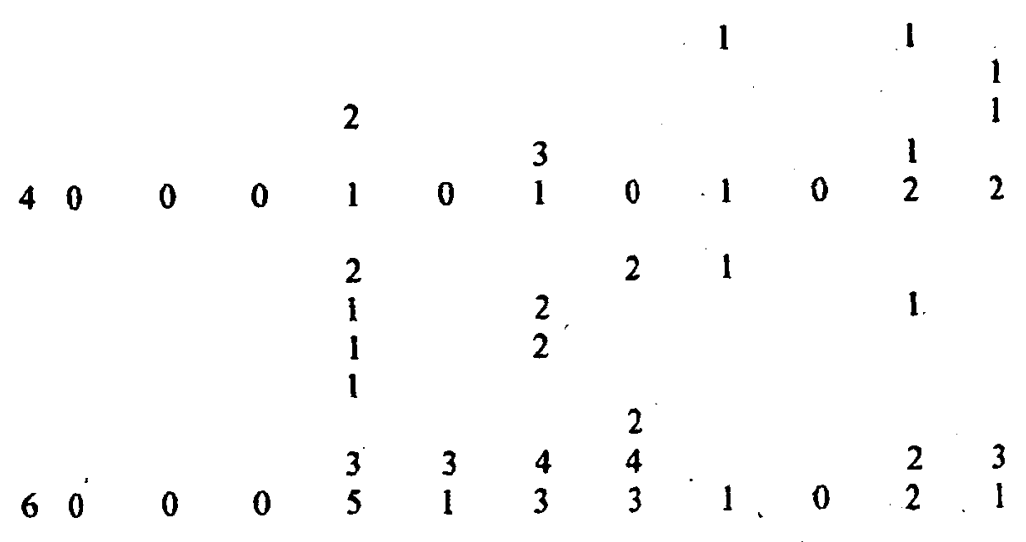

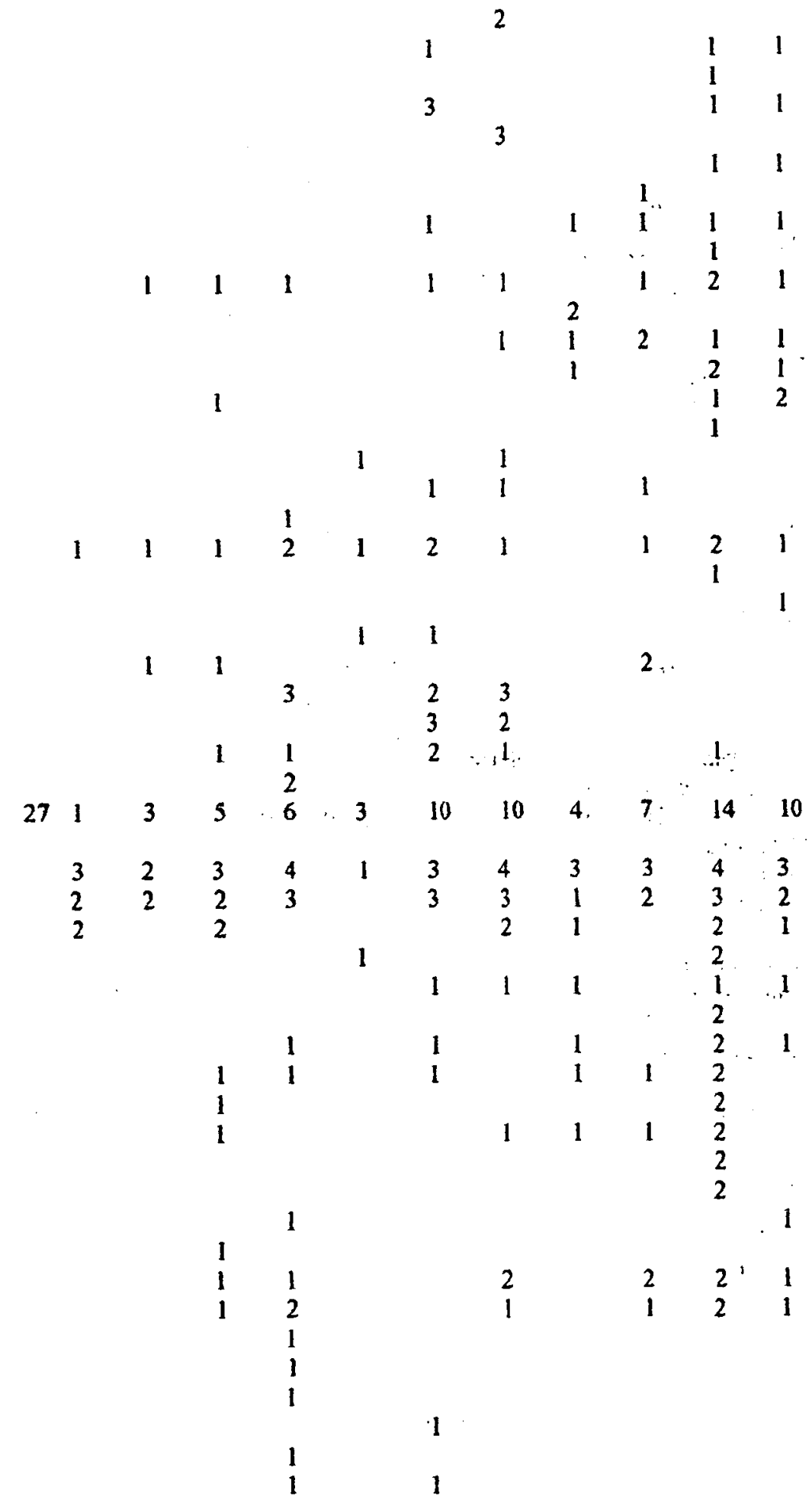


Tableau 2. Suite

Table 2. Continued.

Potamonectes clarki Woll. Potamonectes nemethe Thery. Graptodytes bermondi Guign Graptodytes acqualis Zimm. Graptodytes varius Aubé. Graptodytes ignotus Muls. Graptodytes atlantis Thery. Rhantus pulverosus Steph. Hygrobia tarda Herbst Haliplus lineatocollis March Haliplus mucronatus Steph. Haliplus ruficeps Chevr. Aulonogynus striatus Fab.

Gyrinus substriatus Stephan Hydrobius fuscipes L.

Hydrobius convescus Brullé Helochares lividus Forster

Enochrus bicolor Fab.

Hydrous piceus $\mathrm{L}$.

Hydrophilus caraboldes $\mathrm{L}$.

Berosus affinis Brullé

Coelostoma hispanium Küster

Limnebius furcatus Baudi Helophorus rufipes Bosc

Helophorus alternans Gene Helophorus porculus Bedel

Helophorus oxygonus Bedel

Helophorus viridicollis Stephens

Helophorus elizabetae Angus

Hydrochus angustatus Germar

Hydrochus nitidicollis Muls.

Hydraena regularis Rey

$$
\mathrm{N} \text { taxons }
$$

TRICHOPTERES

Hydropsyche maroccana Navas

Psychomyia pusilla Fab.

Hydroptila angulata Mosely

Hydroptila vectis Curtis

Ecnomus deceptor Mcl

Rhyacophila munda oreina Navas

Chimarra marginata $\mathrm{L}$.

DIPTERES $\mathrm{N}$ taxons

Tipulidae

Simuliidae

Culicidae

Psychodidae

Ceratopogonidae

Dolichopodidae

Chaoboridae

Stratiomyidae

Thaumaleidae

Chironomidae

Tanytarsini

Chironomini

Dixa sp.

Empididae

Syrphidae

Sciomyzidae

Tabanus sp.

$N$ taxons TOTAL

54

2

2

$\begin{array}{llllllll}2 & & & 2 & & & & \\ 1 & & & & & & 2 & \\ 1 & & & & 1 & & 2 & \\ 1 & & & & & & 2 & \\ 1 & & 1 & 2 & & 1 & & \\ 1 & & & 2 & & & & 1 \\ & & 1 & 2 & & 1 & & 1 \\ 3 & 1 & 1 & 2 & 1 & 2 & 2 & 2 \\ 3 & & 2 & 3 & & 2 & & 1 \\ & & & & & & 2 & \end{array}$

1

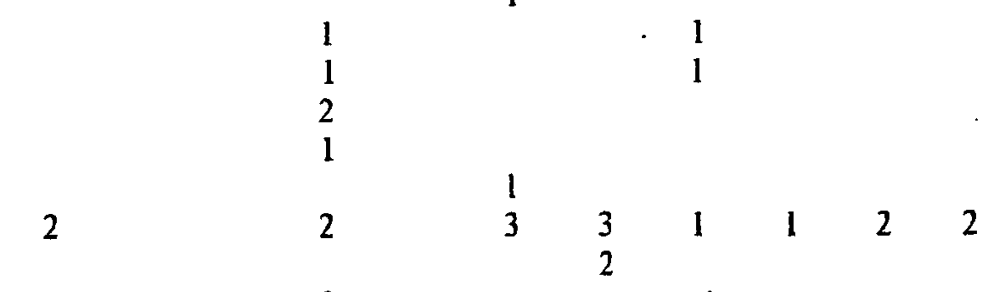

$\begin{array}{llll}3 & 3 & 2 & 1 \\ 1 & 2 & 2 & 3 \\ & 1 & 2 & 1 \\ 1 & & & 3 \\ 3 & 2 & 3 & 3\end{array}$

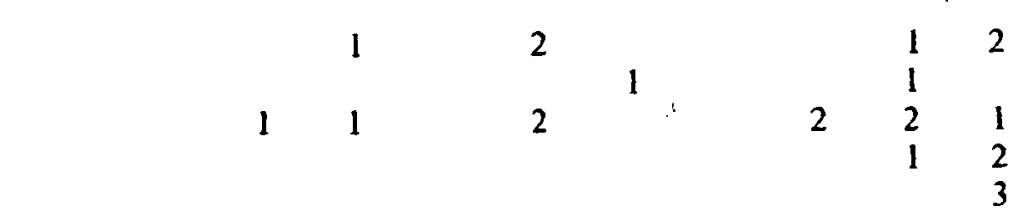

$\begin{array}{lllll}2 & 1 & 2 & 2\end{array}$

$\begin{array}{llllllllllll}7 & 0 & 0 & 1 & 4 & 0 & 3 & 1 & 0 & 1 & 5 & 5\end{array}$

$\begin{array}{llllllllll}2 & 1 & 3 & 2 & 1 & 3 & & 2 & 3 & 2 \\ & & 2 & & & 2 & 3 & 2 & 2 & \end{array}$

$\begin{array}{llllllllll}2 & & 2 & & & & 1 & 2 & & 2 \\ & & 2 & & 1 & 1 & & & & \\ 3 & 1 & 4 & 1 & 1 & 1 \\ & & & & & & & & & \\ & 2 & & & 2 & 1 & 2 & 1 & & \end{array}$

$\begin{array}{llllllllll}3 & 2 & 3 & 2 & 4 & 2 & 2 & 1 & 3 & 2\end{array}$

\begin{tabular}{|c|c|c|c|c|c|c|c|c|c|c|c|c|}
\hline $\begin{array}{l}\text { Tabanus sp. } \\
\mathrm{N} \text { taxons }\end{array}$ & 17 & 1 & $\begin{array}{l}1 \\
4\end{array}$ & 4 & $\begin{array}{l}1 \\
7\end{array}$ & $\begin{array}{l}2 \\
4\end{array}$ & $\begin{array}{l}1 \\
10\end{array}$ & $\begin{array}{l}2 \\
10 \\
\end{array}$ & $\begin{array}{l}2 \\
9 \\
\end{array}$ & $\begin{array}{l}2 \\
7\end{array}$ & $\begin{array}{l}2 \\
8 \\
\end{array}$ & 4 \\
\hline TOTAL & 167 & 11 & 18 & 28 & 74 & 21 & 61 & 65 & 63 & 49 & 83 & 69 \\
\hline
\end{tabular}

Espèces exclusives

\begin{tabular}{lllllllllll}
0 & 0 & 1 & 14 & 1 & 7 & 8 & 5 & 2 & 9 & 9 \\
\hline
\end{tabular}




\subsubsection{Evolution temporelle du peuplement}

Les variations de la richesse spécifique par station au cours du cycle d'étude sont représentées sur la figure 5.

La diversité faunistique des 3 stations temporaires situées à l'amont de l'oued (SSO, SS1, SS2), très faible au début de l'étude, augmente légèrement le mois suivant. A l'issue de la période d'assec, cette diversité, reste relativement faible et constante.

Les stations permanentes ( $\mathrm{S} 1$ à S8) présentent une variabilité du nombre de taxons plus ou moins importante avec un pic d'abondance en juillet dans les stations situées les plus en amont ( $\mathrm{S} 1$ et $\mathrm{S} 3$ ), ou en maijuin 1996 dans les autres stations (S4 à S8). Les conséquences de la crue de fin octobre 1996 sont plus ou moins importantes sur cette diversité en fonction de la situation des stations. Elles sont particulièrement nettes dans la station $\mathrm{S} 1$ où 28 taxons disparaissent durant cette période.

Après la crue de fin octobre 1996, le nombre de taxons qui avait globalement chuté, augmente à partir du mois de décembre dans les stations S1, S6, S7 et S8.
Les autres stations ont été moins affectées et leur diversité reste relativement constante.

\subsubsection{Quelques données autécologiques et écolo- giques}

En plus de celles fournies par ce travail, des informations susceptibles d'expliquer la répartition de certains groupes peuvent être tirées de travaux antérieurs, en particulier ceux de Dakki (1987).

\section{- Mollusques}

Selon Dakki (1987) les genres Melanopsis, Valvata, Limnaea, Bulinus et Pisidium, sont communs dans les sources et les ruisseaux frais du bassin du Sebou. Ce n'est pas le cas pour les sources de cette étude qui n'en possèdent que peu (S2 : Physa acuta et Planorbis planorbis) ou pas (SS0). De plus, les espèces citées par cet auteur se rencontrent essentiellement dans les stations situées à l'aval (exception faite de Limnaea truncatula).

— Ephéméroptères

Elles sont absentes des 3 premières stations. Contrairement à ce qui a été observé dans le bassin du Sebou,
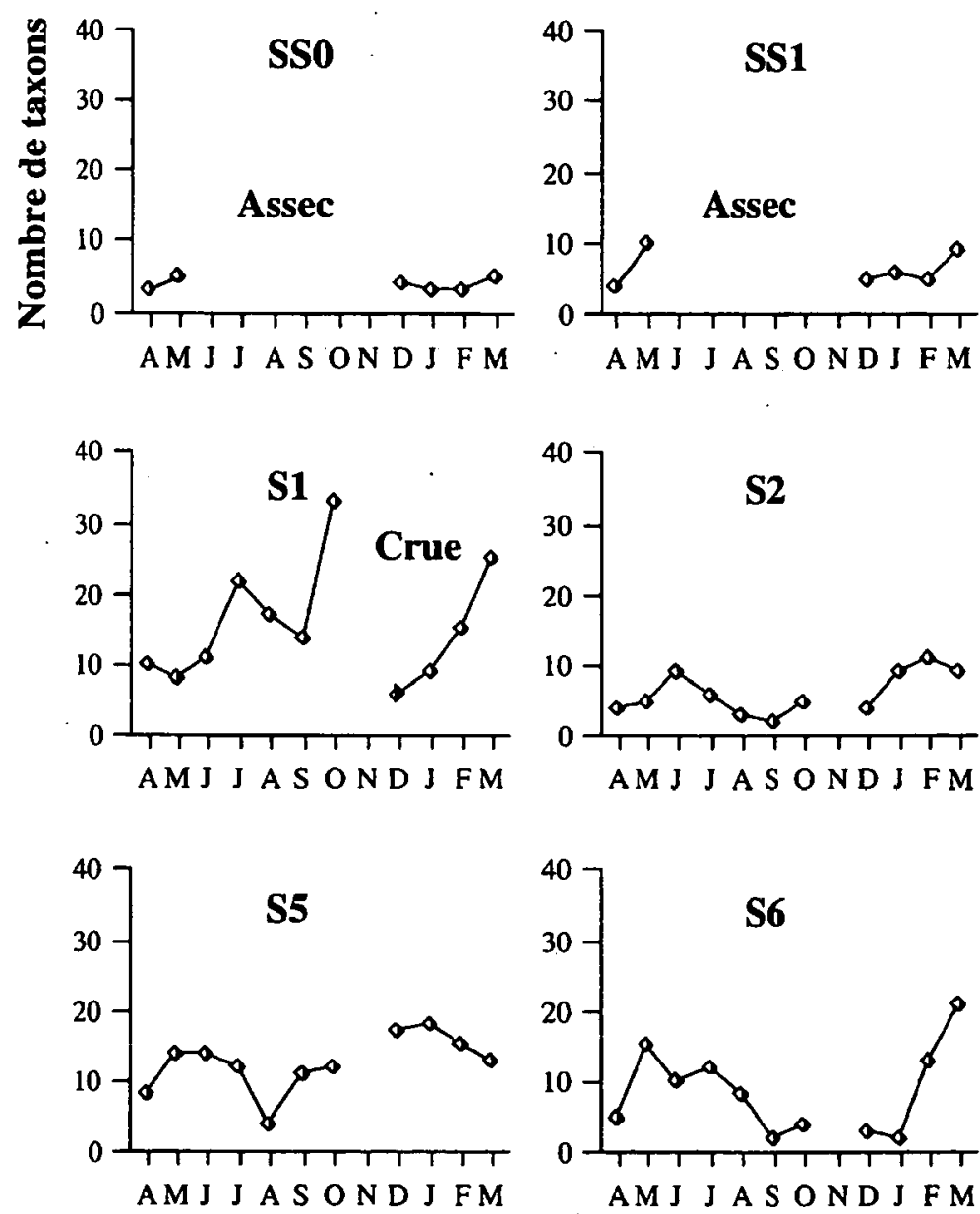
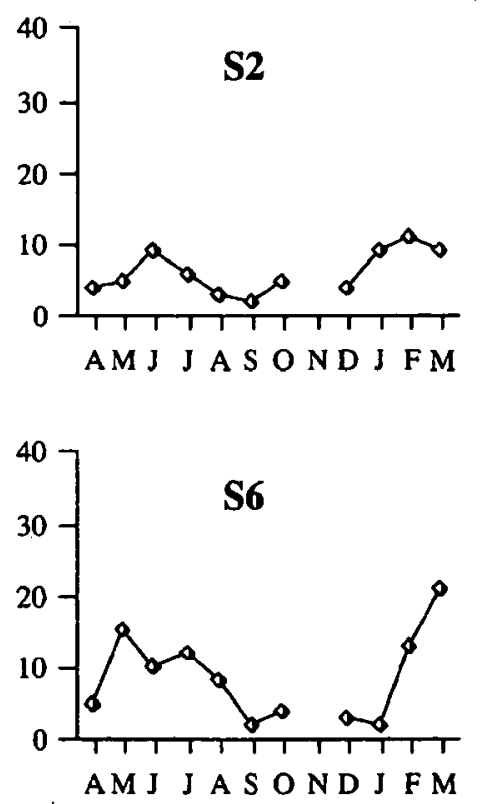
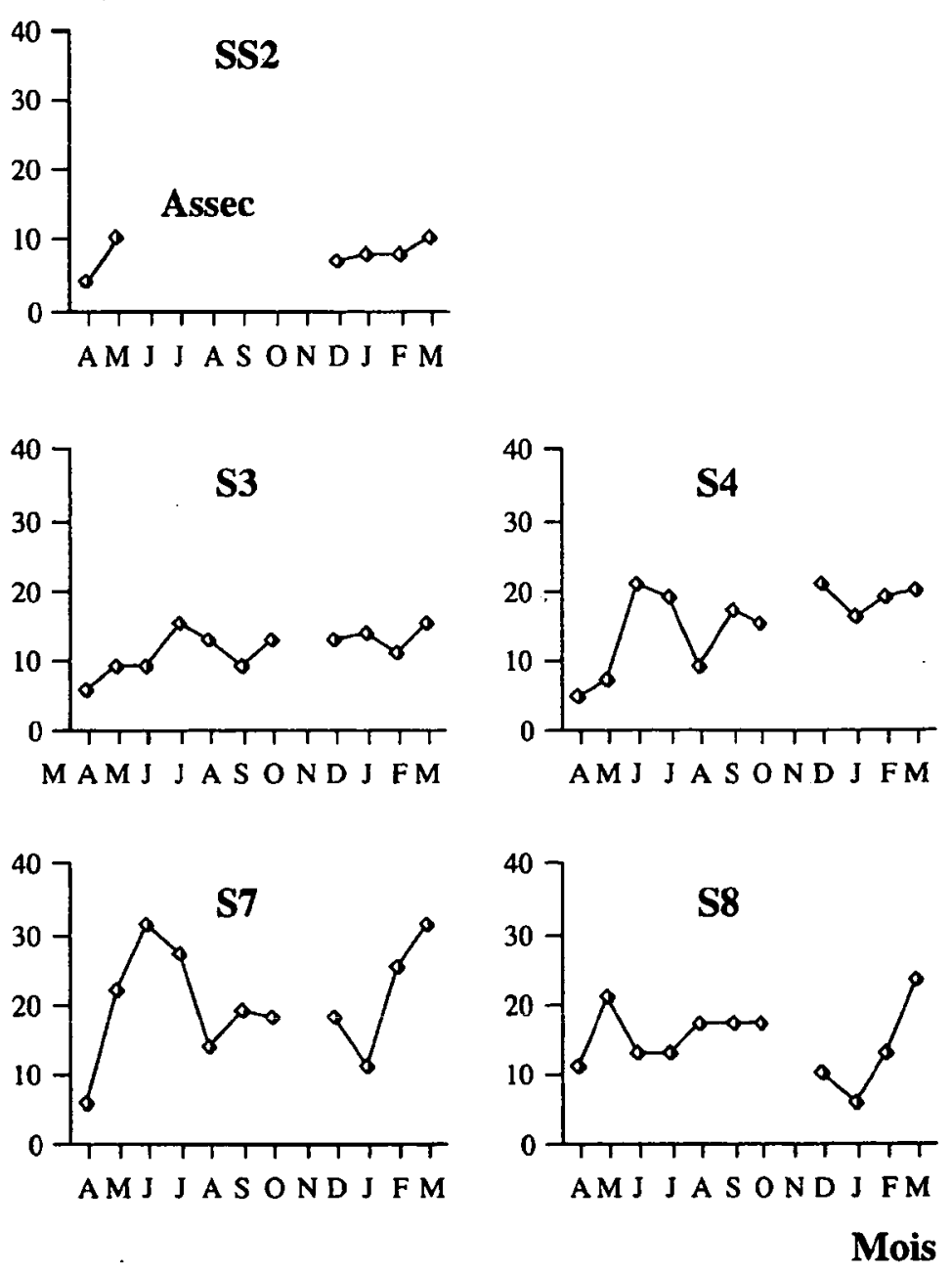

Fig. 5. Evolution temporelle de la richesse spécifique des différentes stations.

Fig. 5. Changes of species richness versus time. 
(Dakki 1987), Potamanthus luteus (Potamanthidae), peuple l'ensemble de la rivière avec des effectifs assez importants.

Trois espèces d'Heptageniidae ont été récoltées : $E c$ dyonurus rothschildi, largement répandue, est relativement thermophile. La seconde, Ecdyonurus forcipula est nettement moins thermophile et plus alticole que la première, ce qui pourrait expliquer en partie son absence dans les stations situées le plus à l'aval. Rhithrogena ayadi, rhéophile et psychrotherme de relativement haute altitude, est naturellement absente des stations situées le plus à l'aval.

Les 2 espèces endémiques d'Oligoneuriidae - Oligoneuriella skoura et Oligoneuriopsis skhounate rencontrées essentiellement dans les stations S4, et S7 (plus $\mathrm{S} 8$ pour $O$. skhounate) ont été recensées dans les rivières rapides, chaudes et tempérées de basse et moyenne altitude. Leur présence est tout à fait normale dans la partie basse de l'oued.

Parmi ces Baetidae, Baetis rhodani est considérée comme ubiquiste et est rencontrée dans les biotopes froids et tempérés de haute et moyenne altitude. Sa présence en quantité importante dans les stations $\mathrm{S} 1$ et S3 confirme ces données.

Ephemera glaucops (Ephemeridae) qui préfère les milieux lénitiques et chauds des rivières, n'est récoltée en petite quantité que dans la station $\mathrm{S} 1$ et dans les deux dernières stations.

Ephemerella ignita (Ephemerellidae) qui vit en général dans des zones semi-temporaires, n'a été récoltée que dans la station 4 .

\section{- Plécoptères}

Seules 4 espèces ont été récoltées dans quelques stations permanentes, ce qui tend à prouver la relative pauvreté spécifique de cet ordre dans le Moyen-Atlas (Dakki 1987). Sur les 4, 2 sont endémiques (B. algirica et $P$. algirica).

\section{- Trichoptères}

Sept espèces appartenant à 6 familles ont été récoltées. Deux sont endémiques : Hydropsyche maroccana et Rhyacophila munda oreina). Une seule espèce ( $H y$ droptila angulata) est présente dans la station temporaire SS2. Il s'agit d'une espèce thermophile de basse et moyenne altitude qui se rencontre dans pratiquement l'ensemble des stations. Les autres espèces, dont la répartition est beaucoup plus réduite, vivent dans des cours d'eau qui se réchauffent en été, et généralement dans les faciès lénitiques.

- Coléoptères
Ils sont présents partout. Certaines espèces d'Agabus et d'Elephorus peuplent les 3 stations supérieures. La station S1 est la plus diversifiée et renferme 9 espèces exclusives sur les 14 dénombrées. Sur les six espèces exclusives récoltées dans la station $\mathrm{S} 3$, trois sont des Hydrocanthares : Hydroporus planus, Graptodytes atlantis, Gyrinus substriatus et deux des Hydrophilides : Hydrochus nitidicollis, Hydrous piceus. Ces espèces se rencontrent fréquemment dans les eaux boueuses et sont parmi les meilleures nageuses de leur groupe (Aouad 1984).

\section{Discussion et conclusion}

Les valeurs des paramètres physico-chimiques sont tout-à-fait comparables à celles trouvées lors d'autres investigations faites au Maroc ou en Algérie ou à celles évoquées par Nisbet \& Verneaux (1970), en particulier pour les stations situées vers les sources karstiques (Gagneur \& Chaoui-Boudghane 1991, Essafi et al. 1993, Chahlaoui et al. 1997, Berrady et al. 2000). Ces paramètres témoignent d'une bonne qualité de l'eau sur tout le cours de cet oued, à l'exception de la station S5 située à l'aval de la ville d'Ifrane, pour laquelle la quantité d'oxygène dissous est particulièrement faible. Toutefois, les teneurs en oxygène dissous aux émergences karstiques SS0 et S2 sont élevées par rapport aux normes des sources karstiques (Nisbet \& Verneaux 1970). Les variations de ces paramètres mésologiques le long de l'oued, selon un continuum depuis la source, mais également en fonction des saisons, en relations étroites avec d'autres facteurs structurant comme l'altitude (Berrahou 1988) ou bien les caractéristiques morphodynamiques (végétation, éclairement, pente...), influent sur la répartition de la faune aquatique.

Il en résulte une originalité faunistique qui se traduit d'abord par la présence d'un peuplement à caractère limnophile indicateur de la bonne qualité de l'eau et dont l'abondance de l'amont vers l'aval est régie par les ressources trophiques et la diversité des habitats. La biodiversité qui en résulte (167 taxons) apparaît importante comparée à celle d'autres cours d'eau marocains du même type. A titre d'exemple, 104 taxons ont été signalés dans un cours d'eau des Beni-Snassen (Berrahou 1988), 130 espèces dans le Tensift (Badri 1985), et seulement 53 espèces dans le réseau de l'assif d'Oukaïmeden (Mohati 1985). Il en est de même pour les oueds de la région de Tlemcen en Algérie où le nombre d'espèces de Coléoptères (10), Hétéroptères (4) et Ephéméroptères (6) (Gagneur \& Chaoui-Boudghane 1991) apparaît faible par rapport à celui de l'oued Tizguit. 
Contrairement aux observations de Glazier (1991) ou de Berrady et al. (2000) sur d'autres cours d'eau de même type, la source karstique SS0 ne renferme que des insectes. Légèrement plus à l'aval, les stations SS1, SS2, puis S2, encore sous l'influence de cette source karstique, présentent quelques taxons autres que les insectes, comme les Annélides et les Mollusques. En revanche, la station S4 qui reçoit aussi des eaux d'origine karstique présente de nombreux taxons autres que les insectes. Ce dernier résultat est en accord avec les hypothèses de Glazier (1991), selon lesquelles la constance relative de paramètres mésologiques - en particulier température et débit - permet l'installation de taxons autres que les insectes au détriment de ceux-ci.

L'augmentation du nombre d'espèces d'avril à mai 1996, dans ces stations situées à l'amont, est due à une période très favorable au développement de la faune. Mais, leur mise à sec a été trop rapide, et a duré trop longtemps, pour permettre l'installation, par la suite, d'un nombre plus important d'espèces, et pour qu'une colonisation importante du milieu puisse se faire lors de la remise en eau. Cette observation corrobore celles faites par Gagneur \& Chaoui-Boudghane (1991), et confirme l'évolution particulière des peuplements des ruisseaux temporaires au cours du temps.

Pour les stations pérennes, à l'exception de S1, la plus riche de l'amont (75 taxons), et de S2 qui est pauvre (21 taxons), vraisemblablement du fait de la proximité de la résurgence, existe un continuum de la diversité de l'amont vers l'aval. Les légères variations de cette richesse spécifique sont essentiellement dues aux confluences avec les sources et aux caractéristiques mésologiques proches des stations, en particulier à l'existence plus ou moins importante de la ripisylve. Cette dernière joue, en effet, un rôle important dans le fonctionnement de l'hydrosystème (source majeüre de mâtié organique, stabilité des berges et diversité d'habitats pour les insectes aquatiques grâce à son réseau racinaire et aux branches qui tombent dans l'eau) et, par conséquent, contribue largement à l'installation d'une faune riche et diversifiée (Maridet et al. 1996, 1997).

La variation̈..longitudinale des valeurs des paramètres mésologiques va aussi pouvoir se traduire par un phénomène de vicariance (sensu Décamps 1968) : remplacement successif, de l'amont vers l'aval, d'espèces plus ou moins sténoèces. Ce phénomène est observé en particulier chez les Ephéméroptères pour lesquels les Heptageniidae rencontrés plutôt à l'amont, sont remplacés par les Oligoneuriidae.
La station S5, à l'aval d'Ifrane, ne semble pas aussi polluée qu'il serait logique de le penser, et les rejets d'origine domestique qui s'y déversent sont certainement rapidement épurés car les valeurs des paramètres physico-chimiques, en dehors de l'oxygène dissous et du $\mathrm{pH}$, ne présentent pas de différences notoires par rapport aux autres stations. C'est ainsi que l'on peut noter la présence d'Helophorus et de Gammarus, organismes qui se rencontrent généralement dans des eaux non polluées.

Ainsi, l'augmentation générale de la biodiversité de ce petit oued peut certainement être expliquée dans le cas de l'augmentation classique en nombre et en amplitude des niches écologiques par suite de l'augmentation de la taille du cours d'eau. La spécialisation qui apparaît maximale au niveau des sources est certainement due à une stabilité thermique plus grande et des apports trophiques moins importants qu'ailleurs.

\section{Remerciements}

Une partie de ce travail a été réalisée grâce à l'Action Intégrée Franco-Marocaine 97/115/SVS. Les auteurs tiennent à remercier pour leur aide à la détermination des différents taxons : Mr Dakki pour les Trichoptères, Mr El Alaoui pour les Coléoptères Hydrocanthares, Mr Fekhaoui pour les Diptères, Mr Gueit pour les Hétéroptères, Mme Guemmouh pour les Odonates. La collection du Muséum de l'Institut Scientifique de Rabat a servi de matériel de référence pour la vérification ou l'identification de certaines espèces.

\section{Travaux cités}

Aguesse P., Dakki M., Gheit A. \& Ramdani M.1982. — Les Hétéroptères aquatiques du Maroc. Inventaire commenté. Bull. Inst. Sc. Rabat, 6: 125-138.

Aouad N. 1984. - Etude écologique des Hydrophiloidae d'eaux stagnantes de la région de Rabat (Coléoptères Palpicornes). Thèse de $3^{\text {ème }}$ cycle, Univ. Paul Sabatier, Toulouse : $112 \mathrm{p}$.

Aouad N. 1991. - Le polymorphisme des insectes aquatiques au Maroc : exemple des Hydrophiloïdea (Coléoptères Palpicornes). Doctorat d'Etat es-Sciences en Ecologie, Fac. Sc. Rabat : 183 p.

Badri A. 1985. - Etude hydrobiologique d'un cours d'eau de plaine en zone semi-aride : Tensift. Impacts des crues sur la biocénose. Thèse de $3^{\text {ème }}$ cycle, Fac. Sc. Marrakech : $134 \mathrm{p}$.

Beauchamp P. 1949. - Nouvelles stations de rhéophiles dans l'Atlas. Bull. Soc. Nat. Maroc, 29 : 251-252.

Benhoussa A., Dakki M., El Agbani M.A. \& Qninba A. 1988. Dynamique et cycle biologique de quelques populations simulidiennes (Diptera-Simuliidae) du Bou Regreg (Plateau Central Marocain). Bull. Inst. Sci., Rabat, 12 : 157-165.

Berrady I., Essafi K. \& Mathieu J. 2000. - Comparative physicochemical and faunal studies of two thermal springs in Sidi-Harazem (Morocco). Ann. Limnol., 36 (4) : 261-274.

Berrahou A. 1988. - Recherches hydrobiologiques sur un cours d'eau des Beni-Snassen (Maroc nord-oriental). Impact des crues sur les macroinvertébrés benthiques. Thèse de $3^{\text {ème }}$ cycle, Fac. Sc. Rabat : $124 \mathrm{p}$.

Chahlaoui A., Cugny P., Lek S., Zaid A. \& Ramdani M. 1997. Analyse quantitative de la qualité de l'eau de l'oued Boufekrane (Maroc). Bull. Soc. Hist. Nat. Toulouse, 133 : 71-76. 
Colo G. 1960-1961. - Contribution à l'étude du Jurassique du Moyen Atlas septentrional. Notes et Mém. Serv. Géol. Maroc, 139 et 139 bis (Atlas) : $226 \mathrm{p}$.

Dakki M. 1979. - Recherches hydrobiologiques sur un cours d'eau du Moyen-Atlas (Maroc). Thèse de $3^{\text {ème }}$ cycle Aix-Marseille III, $126 \mathrm{p}$.

Dakki M. 1985 a. - Sur le choix des données en biotypologie des eaux courantes par l'analyse factorielle des correspondances. Bull. Ecol., 16 (4) : 285-296.

Dakki M. 1985 b. - Biotypologie et gradient thermique spatio-temporels, étude sur un cours d'eau du Moyen-Atlas (Maroc). Bull. Ecol., 17 (2) : 79-85.

Dakki M. 1986 a. - Recherches hydrobiologiques sur le haut Sebou (Moyen-Atlas) : une contribution à la connaissance faunistique, écologique et historique des eaux courantes sud-méditerranéennes. Doctorat d'état es-Sciences en écologie, Fac. Sc. Rabat : $214 \mathrm{p}$.

Dakki M. 1986 b. - Biotypologie et gradient thermique spatio-temporels, étude sur un cours d'eau du Moyen-Atlas (Maroc). Bull. Ecol., 17 (2) : 79-85.

Dakki M. 1987. - Ecosystèmes d'eau courante du haut Sebou (Moyen-Atlas) : études typologiques et analyses écologique et biogéographique des principaux peuplements entomologiques. Travaux de l'Inst. Sc. Chérifien. Série Zoologie, Rabat, 42 : 99 p.

Décamps H. 1968. - Vicariances écologiques chez les Trichoptères des Pyrénées. Ann. Limnol., 4 (1) : 1-50.

El Agbani M.-A., Dakki M. \& Bournaud M. 1992 - Etude typologique du Bou Regreg (Maroc) : les milieux aquatiques et leurs peuplements en macroinvertébrés. Bull. Ecol., 23 (1-2) : 103-113.

Essafi K., Mathieu J. \& Chergui H. 1993. - Les paramètres physico-chimiques en tant que facteurs explicatifs des relations entre les eaux superficielles karstiques et interstitielles de l'oued Zeghzel (Maroc oriental). Mém. Biospéol., XX : 81-89.

Gagneur J. \& Chaoui-Boudghane C. 1991. - Sur le rôle du milieu hyporhéique pendant l'assèchement des oueds de l'Ouest Algérien. Stygologia, 6 (2) : 77-89.

Giudicelli J. \& Dakki M. 1984. — Les sources du Moyen-Atlas et du Rif (Maroc) : faunistique (description de 2 espèces nouvelles de Trichoptères), écologie, intérêt biogéographique. Bijdr. Dierk., $54,1: 83-100$.
Glazier D.S. 1991. - The fauna of North American temperate cold spring : patterns and hypotheses. Freshwat. Biol., 26 : 527-542.

Iablokoff A.K. 1954. - Un mois de recherches écologiques dans le 'Moyen-Atlas. Bull. Soc. Sc. Nat. Phys. Maroc, 34, 1 : 97-128.

Lepiney L. 1961.- Recherches écologiques et biologiques sur les crustacés de deux mares temporaires. Travaux de l'Inst. Sc. Chérifien. Série Zoologie, Rabat, $\mathrm{n}^{\circ} 25: 4$.

Maridet L., Philippe M., Wasson J.G. \& Mathieu J. 1996. - Spatial and temporal distribution of macroinvertebrates and trophic variables within bed sediment of three streams with different vegetal environment and morphology. Arch. Hydrobiol., 136, 1 : 41-64.

Maridet L., Philippe M., Wasson J.G. \& Mathieu J. 1997. — Seasonal dynamics and storage of particulate organic matter in the bed sediment of three streams with contrasted riparian vegetation. In Groundwater Surface water Ecotones. J. Gibert, J. Mathieu, F. Fournier (Eds), Cambridge University Press, 68-74.

Mohati A. 1985. - Recherches hydrobiologiques sur un cours d'eau du Haut Atlas de Marrakech (Maroc) : l'Oued Ourika. Ecologie, biotypologie et impact des activités humaines sur la qualité des eaux. Thèse de $3^{\text {ème }}$ cycle, Fac. Sci. Marrakech : $108 \mathrm{p}$.

Mouna M. 1988. - La bioécologie et l'environnement biologique d'Acleris undulana Walsingham (Lep. Tortricidae), ravageur du cèdre dans le M-A marocain. Doct. es-Sc.Nat. en Zoologie. Univ. Mohamed V, Fac. Sc. Rabat.

Nisbet M. \& Verneaux J. 1970. - Composantes chimiques des eaux courantes. Ann. Limnol., 6 (2) : 161-190.

Rodier J. 1996. - L'analyse de l'eau : eaux naturelles, eaux résiduaires, eau de mer. Edition Dunod Paris : 365 p.

Qninba A., El Agbani M.A., Dakki M. \& Ben Houssa A. 1988. Evolution saisonnière de quelques peuplements d'invertébrés benthiques de l'Oued Bou Regreg (Maroc). Bull. Inst. Sci., Rabat, 12 : 149-156.

Thioulouze J., Chessel D., Dolédec S. \& Olivier J.M. 1997. - ADE$4:$ a multivariate analysis and graphical display software. Statistics \& computing, 7 : 75-83.

Zeryouhi M. 1977. - Hydrogéologie du Moyen-Atlas. In Serv. géol. Maroc (éd.) : Ressources en eau du Maroc, tome 3 : domaines atlasique et sud-atlasique. Notes et mém. Serv. géol. Ma$r o c, \mathrm{n}^{\circ} 231: 67-84$ 